\title{
String cosmological model in the presence of a magnetic flux
}

\author{
Bijan Saha \\ Laboratory of Information Technologies \\ Joint Institute for Nuclear Research, Dubna \\ 141980 Dubna, Moscow region, Russid* \\ Mihai Visinescu \\ Department of Theoretical Physics \\ National Institute for Physics and Nuclear Engineering \\ Magurele, P. O. Box MG-6, \\ RO-077125 Bucharest, Romanid
}

\begin{abstract}
A Bianchi type I string cosmological model in the presence of a magnetic flux is investigated. A few plausible assumptions regarding the parametrization of the cosmic string and magneto-fluid are introduced and some exact analytical solutions are presented.

PACS numbers: 03.65.Pm and 04.20.Ha

Keywords: Spinor field, Bianchi type I (BI) model, Cosmological constant, Magneto-fluid
\end{abstract}

\section{INTRODUCTION}

Since the observation of the current expansion of the Universe which has apparently accelerated in the recent past, the anomalies found in the cosmic microwave background (CMB) and the large structures observations it becomes obvious that a pure Friedmann-Lemaitre-RobertsonWalker (FLRW) cosmology should be amended.

Bianchi type I cosmological models are the simplest anisotropic Universe models playing an important role in understanding essential features of the Universe. In this class of models it is possible to accommodate the presence of cosmic strings. In the last time the string cosmological models have been used in attempts to describe the early Universe and to investigate anisotropic dark energy component including a coupling between dark energy and a perfect fluid (dark matter) [1]. Cosmic strings are one dimensional topological defects associated with spontaneous symmetry breaking in gauge theories. Their presence in the early Universe can be justified in the frame of grand unified theories (GUT).

The object of this paper is to investigate a Bianchi type I string cosmological model in the presence of a magnetic flux. The inclusion of the magnetic field is motivated by the observational cosmology and astrophysics indicating that many subsystems of the Universe possess magnetic fields (see e. g. the reviews [2, 3] and references therein).

In the following section we introduce a system of cosmic string and magnetic field in the Bianchi type I cosmology presenting some of its general features. In Section III we introduce a few

*Electronic address: bijan@jinr.ru; URL: http://www.jinr.ru/ bijan/

${ }^{\dagger}$ Electronic address: mvisin@theory.nipne.ro, URL:http://www.theory.nipne.ro/ mvisin/ 
plausible assumptions usually accepted in the literature and some exact solutions are produced. In the last section we present some conclusions and perspectives.

\section{FUNDAMENTAL EQUATIONS AND GENERAL SOLUTIONS}

We consider the gravitational filed given by an anisotropic Bianchi type I (BI) metric

$$
d s^{2}=a_{0}^{2}\left(d x^{0}\right)^{2}-a_{1}^{2}\left(d x^{1}\right)^{2}-a_{2}^{2}\left(d x^{2}\right)^{2}-a_{3}^{2}\left(d x^{3}\right)^{2},
$$

with $a_{0}=1, x^{0}=c t$ and $c=1$. The metric functions $a_{i}(i=1,2,3)$ are the functions of time $t$ only.

Einstein's gravitational field equation for the BI space-time has the form

$$
\begin{aligned}
\frac{\ddot{a}_{2}}{a_{2}}+\frac{\ddot{a}_{3}}{a_{3}}+\frac{\dot{a}_{2}}{a_{2}} \frac{\dot{a}_{3}}{a_{3}} & =\kappa T_{1}^{1}, \\
\frac{\ddot{a}_{3}}{a_{3}}+\frac{\ddot{a}_{1}}{a_{1}}+\frac{\dot{a}_{3}}{a_{3}} \frac{\dot{a}_{1}}{a_{1}} & =\kappa T_{2}^{2}, \\
\frac{\ddot{a}_{1}}{a_{1}}+\frac{\ddot{a}_{2}}{a_{2}}+\frac{\dot{a}_{1}}{a_{1}} \frac{\dot{a}_{2}}{a_{2}} & =\kappa T_{3}^{3}, \\
\frac{\dot{a}_{1}}{a_{1}} \frac{\dot{a}_{2}}{a_{2}}+\frac{\dot{a}_{2}}{a_{2}} \frac{\dot{a}_{3}}{a_{3}}+\frac{\dot{a}_{3}}{a_{3}} \frac{\dot{a}_{1}}{a_{1}} & =\kappa T_{0}^{0} .
\end{aligned}
$$

Here $\kappa$ is the Einstein gravitational constant and over-dot means differentiation with respect to $t$. The energy momentum tensor for a system of cosmic string and magnetic field in a comoving coordinate is given by

$$
T_{\mu(m)}^{v}=\rho u_{\mu} u^{v}-\lambda x_{\mu} x^{v}+E_{\mu}^{v},
$$

where $\rho$ is the rest energy density of strings with massive particles attached to them and can be expressed as $\rho=\rho_{p}+\lambda$, where $\rho_{p}$ is the rest energy density of the particles attached to the strings and $\lambda$ is the tension density of the system of strings [4, 5, 6], which may be positive or negative. Here $u_{i}$ is the four velocity and $x_{i}$ is the direction of the string, obeying the relation

$$
u_{i} u^{i}=-x_{i} x^{i}=1, \quad u_{i} x^{i}=0 .
$$

In (2.3) $E_{\mu v}$ is the electromagnetic field given by Lichnerowich [7]

$$
E_{\mu}^{v}=\bar{\mu}\left[|h|^{2}\left(u_{\mu} u^{v}-\frac{1}{2} \delta_{\mu}^{v}\right)-h_{\mu} h^{v}\right] .
$$

Here $\bar{\mu}$ is a constant characteristic of the medium and called the magnetic permeability. Typically $\bar{\mu}$ differs from unity only by a few parts in $10^{5}(\bar{\mu}>1$ for paramagnetic substances and $\bar{\mu}<1$ for diamagnetic). In (2.5) $h_{\mu}$ is the magnetic flux vector defined by

$$
h_{\mu}=\frac{1}{\bar{\mu}} * F_{v \mu} u^{v},
$$

where $* F_{\mu \nu}$ is the dual electromagnetic field tensor defined as

$$
* F_{\mu v}=\frac{\sqrt{-g}}{2} \varepsilon_{\mu v \alpha \beta} F^{\alpha \beta} .
$$


Here $F^{\alpha \beta}$ is the electromagnetic field tensor and $\varepsilon_{\mu v \alpha \beta}$ is the totally anti-symmetric Levi-Civita tensor with $\varepsilon_{0123}=+1$. Here the co-moving coordinates are taken to be $u^{0}=1, u^{1}=u^{2}=u^{3}=$ 0 . We choose the incident magnetic field to be in the direction of $x$-axis so that the magnetic flux vector has only one nontrivial component, namely $h_{1} \neq 0$. In view of the aforementioned assumption from (2.6) one obtains $F_{12}=F_{13}=0$. We also assume that the conductivity of the fluid is infinite. This leads to $F_{01}=F_{02}=F_{03}=0$. Thus We have only one non-vanishing component of $F_{\mu \nu}$ which is $F_{23}$. Then from the first set of Maxwell equation

$$
F_{\mu v ; \beta}+F_{v \beta ; \mu}+F_{\beta \mu ; v}=0
$$

where the semicolon stands for covariant derivative, one finds

$$
F_{23}=\mathcal{J}, \quad \mathcal{J}=\text { const. }
$$

Then from (2.6) in account of (2.7) one finds

$$
h_{1}=\frac{a_{1} \mathcal{J}}{\bar{\mu} a_{2} a_{3}} .
$$

Finally, for $E_{\mu}^{v}$ one finds the following non-trivial components

$$
E_{0}^{0}=E_{1}^{1}=-E_{2}^{2}=-E_{3}^{3}=\frac{\mathcal{J}^{2}}{2 \bar{\mu} a_{2}^{2} a_{3}^{2}} .
$$

Taking the string along $x^{1}$ direction and using co-moving coordinates we have the following components of energy momentum tensor [8]:

$$
\begin{aligned}
& T_{0}^{0}=\rho+\frac{\mathcal{J}^{2}}{2 \bar{\mu}} \frac{a_{1}^{2}}{\tau^{2}}, \\
& T_{1}^{1}=\lambda+\frac{\mathcal{J}^{2}}{2 \bar{\mu}} \frac{a_{1}^{2}}{\tau^{2}}, \\
& T_{2}^{2}=-\frac{\mathcal{J}^{2}}{2 \bar{\mu}} \frac{a_{1}^{2}}{\tau^{2}}, \\
& T_{3}^{3}=-\frac{\mathcal{J}^{2}}{2 \bar{\mu}} \frac{a_{1}^{2}}{\tau^{2}},
\end{aligned}
$$

where we used the definition

$$
\tau=a_{1} a_{2} a_{3} .
$$

It is indeed the volume scale of the BI space-time, i.e., $\tau=\sqrt{-g}$ [9].

In view of $T_{2}^{2}=T_{3}^{3}$ from (2.2b), (2.2c) one finds

$$
a_{2}=a_{3} D \exp \left(X \int \frac{d t}{\tau}\right)
$$

with $D$ and $X$ being integration constants. Due to anisotropy of the source filed, in order to solve the remaining Einstein equation we have to impose some additional conditions. Here we give two different conditions. It can be shown that the metric functions can be expressed in terms of $\tau$. So 
let us first derive the equation for $\tau$. Summation of Einstein Eqs. (2.2a), (2.2b), (2.2c) and 3 times (2.2d) gives

$$
\frac{\ddot{\tau}}{\tau}=\frac{3}{2} \kappa\left(\rho+\lambda+\frac{2 \mathcal{J}^{2}}{3 \bar{\mu}} \frac{a_{1}^{2}}{\tau^{2}}\right)
$$

Let us demand the energy-momentum to be conserved, i.e., $T_{\mu ; v}^{v}=0$, which in our case takes the form

$$
\frac{1}{\tau} \frac{d}{d t}\left(\tau T_{0}^{0}\right)-\frac{\dot{a}_{1}}{a_{1}} T_{1}^{1}-\frac{\dot{a}_{2}}{a_{2}} T_{2}^{2}-\frac{\dot{a}_{3}}{a_{3}} T_{3}^{3}=0 .
$$

After a little manipulation from (2.16) one obtains

$$
\dot{\rho}+\frac{\dot{\tau}}{\tau} \rho-\frac{\dot{a}_{1}}{a_{1}} \lambda=0
$$

\section{SOME EXAMPLES AND EXPLICIT SOLUTIONS}

In the literature there exists a number of relations between $\rho$ and $\lambda$, the simplest one being a proportionality relation:

$$
\rho=\alpha \lambda
$$

with the most usual choices of the constant $\alpha$

$$
\alpha= \begin{cases}1 & \text { geometric string } \\ 1+\omega & \omega \geq 0, \quad p \text { string or Takabayasi string } \\ -1 & \text { Reddy string }\end{cases}
$$

As it was mentioned earlier, to solve the Einstein equations completely, we need to impose some additional conditions. Here we consider the following two.

\section{A. Case 1}

This condition was first used by Bali [10]. Following him let us assume that the expansion scalar $(\theta)$ in the model is proportional to the eigenvalue $\sigma_{1}^{1}$ of the shear tensor $\sigma_{\mu}^{\nu}$. For the BI space-time we have

$$
\begin{aligned}
\theta & =\frac{\dot{a}_{1}}{a_{1}}+\frac{\dot{a}_{2}}{a_{2}}+\frac{\dot{a}_{3}}{a_{3}} \\
\sigma_{1}^{1} & =-\frac{1}{3}\left(4 \frac{\dot{a}_{1}}{a_{1}}+\frac{\dot{a}_{2}}{a_{2}}+\frac{\dot{a}_{3}}{a_{3}}\right) .
\end{aligned}
$$

Writing the aforementioned condition as

$$
\theta=3 n \sigma_{1}^{1}
$$

one comes to the following relation

$$
a_{1}=Z\left(a_{2} a_{3}\right)^{N}
$$

where $N=-(n+1) /(4 n+1)$ being the proportionality constant and $Z$ is the integration constant. 
From (2.14) and (3.6) after some manipulation for the metric functions one finds [8]

$$
\begin{aligned}
& a_{1}=Z^{1 /(N+1)} \tau^{N /(N+1)}, \\
& a_{2}=\sqrt{D}\left(\frac{\tau}{Z}\right)^{1 / 2(N+1)} \exp \left[\frac{X}{2} \int \frac{d t}{\tau}\right] \\
& a_{3}=\frac{1}{\sqrt{D}}\left(\frac{\tau}{Z}\right)^{1 / 2(N+1)} \exp \left[-\frac{X}{2} \int \frac{d t}{\tau}\right] .
\end{aligned}
$$

In this case Eq. (2.17) takes the form

$$
\dot{\rho}+\left(\rho-\frac{N}{N+1} \lambda\right) \frac{\dot{\tau}}{\tau}=0
$$

Eq. (2.15) now reads

$$
\ddot{\tau}=\frac{3}{2} \kappa(\rho+\lambda) \tau+X \tau^{(N-1) /(N+1)}, \quad \text { where } \quad X=\kappa \frac{\mathcal{J}^{2}}{\bar{\mu}} Z^{2 /(N+1)} .
$$

We will see later, the right hand side of (3.9) is the function of $\tau$, hence can be written as

$$
\ddot{\tau}=\mathcal{F}(\tau) .
$$

Eq. (3.10) admits first integral which can be written as

$$
\dot{\tau}=\sqrt{2[\mathcal{E}-U(\tau)]}
$$

with

$$
\mathcal{U}(\tau)=-\int \mathcal{F}(\tau) d \tau
$$

The expression (3.12) can be viewed as potential, while $\mathcal{E}$ as energy level. A detailed analysis of this mechanism can be seen in, e.g., [11].

Let us now study Eqs. (3.8) and (3.9) for different equations of state. Assuming the relation (3.1) between the pressure of the perfect fluid $\rho$ and the tension density $\lambda$ from Eq. (3.8) one finds

$$
\frac{\dot{\rho}}{\rho}=\left(\frac{N}{\alpha(N+1)}-1\right) \frac{\dot{\tau}}{\tau}
$$

with the solution

$$
\rho=C_{0} \tau^{\frac{N}{\alpha(N+1)}-1}
$$

while the equation for $\tau$ reads

$$
\ddot{\tau}=\frac{3}{2} \kappa C_{0}\left(1+\frac{1}{\alpha}\right) \tau^{\frac{N}{\alpha(N+1)}}+X \tau^{\frac{N-1}{N+1}} .
$$

This equation can be set in the following form

$$
\dot{\tau}=\sqrt{\frac{3 \kappa C_{0}(\alpha+1)(N+1)}{N+\alpha(N+1)} \tau^{1+N / \alpha(N+1)}+\frac{X(N+1)}{N} \tau^{2 N /(N+1)}+\varepsilon_{0}}
$$

where $\varepsilon_{0}$ is the integration constant and is related to $\mathcal{E}$ as $\varepsilon_{0}=2 \mathcal{E}$. 


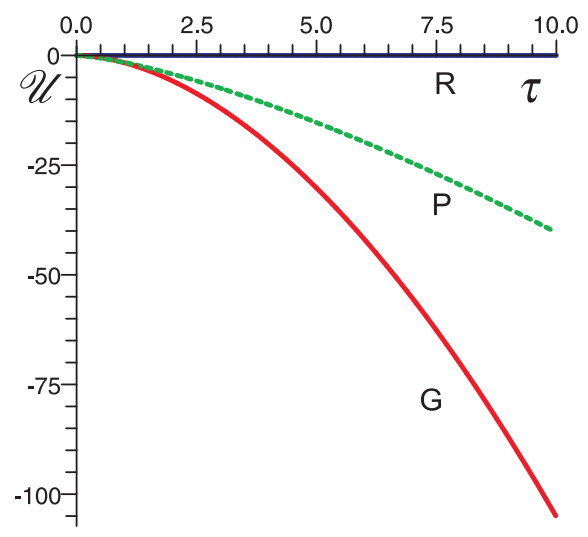

FIG. 1: Potential corresponding to the different equations of state in absence of a magnetic field.

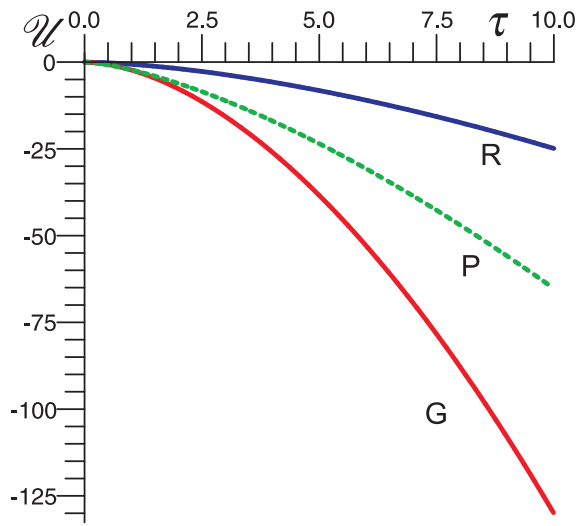

FIG. 3: Potential corresponding to the different equations of state in presence of a magnetic field.

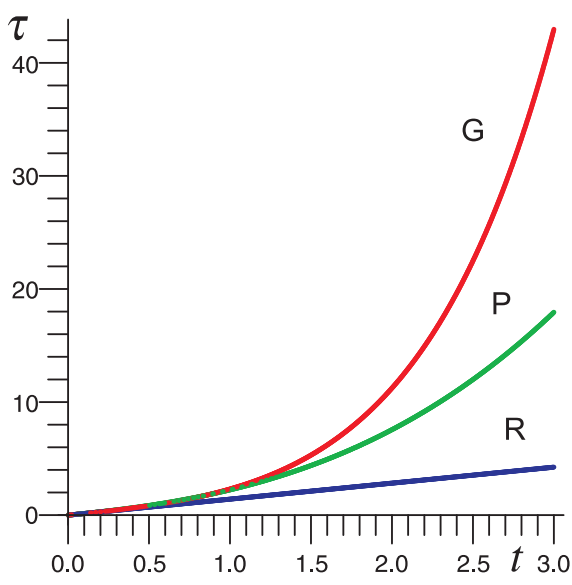

FIG. 2: Evolution of the Universe for different equations of state in absence of a magnetic field.

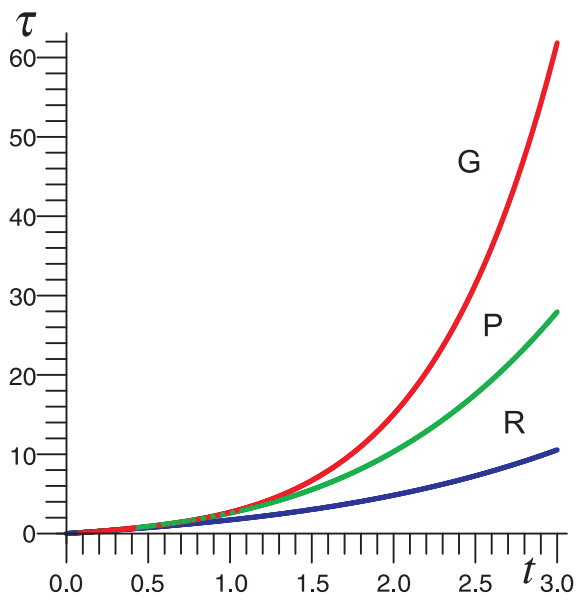

FIG. 4: Evolution of the Universe for different equations of state in presence of a magnetic field.

In Figs. 1 and 3 we have illustrated the potential corresponding to the different equations of state without and with the magnetic field, respectively. The Figs. 2 and 4 show the evolution of $\tau$ for different cases. Here "G", "P" and "R" stand for geometric string, $p$ string and Reddy string, respectively. The reason to illustrate figures with and without magnetic field is to show the role of magnetic field. As one sees, in all cases $\tau$ might be zero at the initial stage of evolution, thus giving rise to the initial singularity in one hand, $\tau$ is not bound from above which means in all three cases we have ever expanding Universe. But introduction of magnetic field into the system results in rapid growth of $\tau$. In numerical analysis we used the following value for the problem parameters: $\kappa=1, \omega=1, N=4, Z=1$. In case of string only we set $\mathcal{J}=0$, otherwise $\mathcal{J}=1$. For magnetic permeability we choose $\bar{\mu}=1.00001$ and $\bar{\mu}=0.99999$, respectively. Since it doesn't make any significant change in the behavior of $\tau$, we illustrate only case with $\bar{\mu}=1.00001$. The 
initial value for $\tau$ is taken to be $\tau(0)=0.0001$ and corresponding first derivative is calculated from (3.16) at $\mathcal{E}_{0}=1$.

\section{B. Case 2}

The second case was proposed by Barrow [12]. Here we first introduce the generalized Hubble parameter

$$
H=\frac{1}{3}\left(\frac{\dot{a}_{1}}{a_{1}}+\frac{\dot{a}_{2}}{a_{2}}+\frac{\dot{a}_{3}}{a_{3}}\right)=\frac{1}{3} \frac{\dot{\tau}}{\tau},
$$

and two relative shear anisotropy parameters by

$$
\begin{aligned}
R & =\frac{1}{H}\left(\frac{\dot{a}_{1}}{a_{1}}-\frac{\dot{a}_{2}}{a_{2}}\right), \\
S & =\frac{1}{H}\left(\frac{\dot{a}_{1}}{a_{1}}-\frac{\dot{a}_{3}}{a_{3}}\right) .
\end{aligned}
$$

When $R=S=0$ the universe will be the isotropic flat Friedman universe. If one sets $R=$ const., then in view of (3.17) one finds,

$$
a_{1}=D_{0} a_{2} \tau^{R / 3} .
$$

Then, in view of (2.13), (2.14) we find the following expressions for metric functions

$$
\begin{aligned}
& a_{1}=\left(D_{0}^{2} D\right)^{1 / 3} \tau^{(3+2 R) / 9} e^{\frac{X}{3} \int \frac{d t}{\tau}}, \\
& a_{2}=\left(D_{0}^{-1} D\right)^{1 / 3} \tau^{(3-R) / 9} e^{\frac{X}{3} \int \frac{d t}{\tau}} \\
& a_{3}=\left(D_{0} D^{2}\right)^{-1 / 3} \tau^{(3-R) / 9} e^{\frac{-2 X}{3} \int \frac{d t}{\tau}} .
\end{aligned}
$$

In this case Eq. (2.17) takes the form

$$
\dot{\rho}+\left(\rho-\frac{3+2 R}{9} \lambda\right) \frac{\dot{\tau}}{\tau}-\frac{X}{3 \tau} \lambda=0,
$$

whereas, for $\tau$ in this case we have

$$
\frac{\ddot{\tau}}{\tau}=\frac{3}{2} \kappa\left(\rho+\lambda+\frac{2 \mathcal{J}^{2}}{3 \bar{\mu}}\left(D_{0}^{2} D\right)^{2 / 3} \tau^{(2 R-15) / 9} e^{\frac{2 X}{3} \int \frac{d t}{\tau}}\right) .
$$

Comparing this equation with the corresponding one (3.15) from Case 1, it results that in Case 2 the equation of evolution for $\tau$ is more intricate. Indeed Eq. (3.22) involves the function $\tau$ in an integral and needs further study. A detailed analysis is presently under investigation and will be reported in a forthcoming paper [13].

In the present paper we confine ourselves to a preliminary task considering this equation in an asymptotic regime, for large $t$. To be aware of possible asymptotic behavior of $\rho$ and $\tau$ for large $t$, let us assume again relation (3.1) and Eq. (3.21) becomes

$$
\frac{\dot{\rho}}{\rho}=\left(\frac{3+2 R}{9 \alpha}-1\right) \frac{\dot{\tau}}{\tau}+\frac{X}{3 \alpha} \frac{1}{\tau},
$$

which shows that both sides of this equation are the same function of time. As in the Case 1 Eq. (3.23) allows a behavior of the pressure of the perfect fluid $\rho$ decreasing in time as an inverse power of $t$ :

$$
\rho=C_{1} t^{-\beta}
$$


$C_{1}$ and $\beta \geq 0$ being some constants. Correspondingly, for $\tau$ we get the equation

$$
\left(\frac{3+2 R}{9 \alpha}-1\right) \dot{\tau}+\frac{\beta}{t} \tau+\frac{X}{3 \alpha}=0
$$

with the solution

$$
\tau=-\frac{3 X}{9 \alpha \beta+3+2 R-9 \alpha} t+C_{2} t^{-\frac{9 \alpha \beta}{3+2 R-9 \alpha}} .
$$

It is interesting to observe that Eq. (3.23) allows even an exponential behavior of $\rho$ for large $t$ :

$$
\rho=C_{3} e^{-\gamma t}
$$

which means that the both sides of Eq. (3.23) are a negative constant $(-\gamma)$. The corresponding behavior of $\tau$ from Eq. (3.23) is

$$
\tau=-\frac{X}{3 \alpha \gamma}+C_{4} e^{-\frac{9 \alpha \gamma}{3+2 R-9 \alpha} t}
$$

with $C_{4}$ another arbitrary constant.

The full study of Eq. (3.22), which contains the parameters characterizing the cosmic string and magnetic field, for large $t$ using the asymptotic behaviors (3.24), (3.25) and (3.27), (3.28) will be presented elsewhere [13].

\section{CONCLUSIONS}

In the present paper we investigated in the frame of Bianchi type I models a string cosmological model in the presence of a magnetic field. We used some tractable assumptions concerning the parameters entering the model.

In the case of a proportionality between the trace of the expansion tensor $\theta$ and the eigenvalue $\sigma_{1}^{1}$ of the shear tensor we are able to get explicit analytic solutions. Setting the relative anisotropy parameter $R=$ const the model is more involved, but sufficiently interesting to deserve further study.

\section{Acknowledgments}

This work is supported in part by a joint Romanian-LIT, JINR, Dubna Research Project, theme no. 05-6-1060-2005/2010.

[1] Koivisto T. and Mota D.F., Phys. Lett. B, 644, 104 (2007); Koivisto T. and Mota D.F. , Phys. Rev. D, 75, 023518 (2007).

[2] Grasso D. and Rubinstein H.R., Phys. Rep., 348, 163 (2001).

[3] Widrow L.M., Rev. Mod. Phys., 74, 775 (2002).

[4] Letelier, P.S., Phys. Rev. D, 28, 1424 (1983).

[5] Pradhan A., Yadav A.K., Singh R.P. and Singh V.K., Astrophys. Space Sci., 312, 145 (2007).

[6] Khadekar G.S., Tade S.D., Astrophys. Space Sci. 310, 47 (2007)]. 
[7] Lichnerowicz A., Relativistic Hydrodynamics and Magnetohydrodynamics, (Benjamin, New York, 1967).

[8] Saha Bijan, J. Astrophys. Space Sci. 2005. 299 No. 1, 149-158, (2005).

[9] Saha Bijan, Phys. Rev. D. 64 123501. (2001).

[10] Bali R. Int. J. Theor. Phys. 25, 755 (1986).

[11] Bijan Saha and Todor Boyadjiev, Phys. Rev. D 69, 124010, (2004) [arXiv: gr-qc/0311045].

[12] Barrow J.D., Phys. Rev. D. 557451 (1997).

[13] Saha B. and Visinescu M., in preparation. 